\title{
Indications, fécondance et pronostic de fertilité des phénotypes pathologiques en microscopie électronique
}

\author{
Valérie MITCHELL, Marie-Claire PEERS, Carole MACHETTI, Maryse LEROY, François MARCELLI \\ Biologie de la Reproduction-Spermiologie, Gynécologie Endocrinienne et Médecine de la Reproduction, \\ Andrologie, CHRU Lille
}

\section{INTRODUCTION}

La fécondation chez les mammifères correspond à une série d'évènements en chaîne qui conduit à l'union des génomes mâles et femelles, l'activation de l'ovocyte et l'initiation du cycle cellulaire embryonnaire. Caractériser précisément les anomalies structurales du spermatozoïde c'est comprendre son fonctionnement et par là même les mécanismes défectueux qui conduisent à évaluer sa capacité ou son incapacité à fertiliser. Cette connaissance suggère alors les stratégies pour permettre la fertilisation avec les " outils " de la procréation dont nous disposons actuellement.

Les principaux phénotypes pathologiques homogènes du spermatozoïde mis en évidence en microscopie électronique peuvent concerner des anomalies de la tête (syndrome des spermatozoïdes microcéphales sans acrosome à tête ronde ou globozoospermie), de la pièce connective (syndrome des spermatozoïdes décapités ou «pinheads»), ou du flagelle avec les dysplasies de la gaine fibreuse (DFS) et les dyskinésies ciliaires primitives (DCP). Bien que l'expression de ces phénotypes soit variable, ils sont toujours rares (incidence < $1 \%$ ) et, la plupart du temps, sévères en terme de potentiel de fertilité. Pourtant très différents sur le plan morphologique et physiopathologique, ils présentent une caractéristique commune : les altérations touchent la totalité ou presque des spermatozoïdes. Ainsi on peut parler de phénotypes homogènes ou monomorphes.

Les structures et composants du spermatozoïde ont un rôle différent et spécifique dans la fertilisation, l'implantation et le développement de l'embryon. La fécondan- ce et le pronostic de fertilité de ces phénotypes pathologiques nécessitent donc de connaître la ou les structure(s) impliquée(s) dans les altérations observées. Dans la globozoospermie, il s'agit principalement de l'absence de l'acrosome dans la forme complète du phénotype, le centrosome/centrioles dans le syndrome des spermatozoïdes décapités, l'axonème/structures périaxonémales dans les DFS et DCP. L'acrosome intervient essentiellement dans la reconnaissance gamétique et la pénétration du spermatozoïde au travers de la zone pellucide. Le centrosome initie le développement embryonnaire en participant à la formation de l'aster, à l'apposition des pronuclei, à la ségrégation des chromosomes, et à la 1ère division méiotique. Quant au flagelle, sa mobilité est absolument requise lors des premières étapes de la fécondation.

Par conséquent, l'altération de l'un de ces constituants génère par évidence un défaut de l'une ou l'autre étape de la fécondation. Ainsi, la conception naturelle et la fécondation assistée in vivo et in vitro (FIV classique) sont compromises dans tous ces phénotypes. Ce dernier concept est acquis mais n'est pas si simple, puisque on pourrait dès lors penser que la micromanipulation du spermatozoïde altéré et son introduction dans l'ovocyte (ICSI) est un traitement efficace palliant

Correspondance

Dr Valérie MITCHELL - Laboratoire de Spermiologie, Hôpital A. Calmette, Bd du Pr. J. Leclercq, 59037 Lille cedex Tel 0320445485 - Fax 0320445932 - Email v-mitchell@chru-lille.fr 
l'absence de fécondance in vivo et in vitro du spermatozoïde atteint. Cependant, les anomalies structurales de certains organites du spermatozoïde peuvent être associées à des altérations d'autres constituants tels que la ségrégation méïotique, la structure de la chromatine et de I'ADN.

\section{LES SPERMATOZOÏDES À TÊTE RONDE SANS ACROSOME (GLOBOZOOSPERMIE)}

Ce syndrome a été décrit pour la première fois en 1965 et étudié en microscopie électronique en 1971 par Schirren et al. [9]. Plusieurs mécanismes au cours de la spermiogenèse sont altérés dans la globozoospermie : la formation de l'acrosome et l'élongation du noyau. A côté du phénotype initial, plusieurs formes ont été décrites. Excepté dans de très rares cas, les hommes présentant ce syndrome sont spontanément infertiles. L'absence de l'acrosome est considérée comme la cause de l'inaptitude à féconder l'ovocyte. Alors que les spermatozoïdes à tête ronde sont capables de traverser le mucus cervical, ils ne peuvent pas féconder les ovocytes : ces spermatozoïdes ne pénètrent pas la corona radiata et ne se fixent pas à la zone pellucide. Par conséquent, la micromanipulation et l'injection des spermatozoïdes dans l'ovocyte sont une solution de traitement de l'infertilité de ces hommes. La première grossesse en ICSI avec des spermatozoïdes à tête ronde a été rapportée en 1994 [5]. Plusieurs cas avec des grossesses menées à terme ont suivis [revue dans 3].

A la lumière de ces exemples, il est clair maintenant que la capacité de fécondation des spermatozoïdes à tête ronde sans acrosome est variable, allant de l'échec total à un taux de fécondation normal, et ne peut être prédite même avec les résultats de l'observation des spermatozoïdes en microscopie électronique. Par conséquent, la stratégie actuelle dans la globozoospermie est de proposer l'ICSI à ces couples. II semblerait qu'une activation in vitro des ovocytes avant la micro-injection améliore les taux de fécondation et de grossesse [8]. En effet, un ou plusieurs facteurs spermatiques d'activation de l'ovocyte pourraient faire défaut ou être dérégulés dans les spermatozoïdes à tête ronde sans acrosome. Des modèles animaux montrent que le centrosome pourrait être altéré également car la formation de l'aster est perturbée dans ces spermatozoïdes.

Une origine génétique à la globozoospermie est suspectée, car plusieurs cas familiaux et un certain degré de consanguinité sont constatés. Plusieurs gènes candidats potentiellement impliqués sont étudiés, en particulier ceux impliqués dans la formation de l'appareil de Golgi et du cytosquelette. Les protéines impliquées dans la physiopathologie de ce syndrome ne sont pas identifiées, ce qui ne permet pas aujourd'hui de cibler les gènes candidats.

\section{LES SPERMATOZOÏDES DÉCAPITÉS (PINHEADS)}

Ce syndrome est très rare. II correspond à des anomalies de l'attachement du flagelle à la tête, avec des phénotypes variables : de l'observation de la quasi-totalité des spermatozoïdes décapités lors du spermogramme, jusqu'à l'observation de la fragilité de l'attachement têteflagelle au moment de la micromanipulation du spermatozoïde avec un spermogramme normal. En microscopie électronique, la cause principale est l'absence de plaque basale, plus ou moins associée à des altérations des microtubules et du centrosome. L'origine des altérations est probablement testiculaire au cours de la spermatogenèse.

Les patients atteints de ce syndrome présentent une stérilité primaire sans autre désordre andrologique, même si les flagelles isolés sont mobiles et pénètrent le mucus cervical. Le seul traitement est la fécondation assistée avec micromanipulation des spermatozoïdes dans l'ovocyte. Cependant le succès de l'ICSI apparaît conditionné par un positionnement correct de la pièce intermédiaire proximale à la tête dans l'ovocyte pour obtenir le clivage et la ségrégation des chromosomes. L'évolutivité même des embryons obtenus en ICSI semble compromise avec la distribution anormale des chromosomes entre les blastomères entraînant la dégénération des embryons avant l'implantation. II y aurait donc un intérêt dans ce syndrome à pousser l'évolution des embryons en blastocystes.

La micro-injection de spermatozoïdes présentant ce phénotype provenant de 2 hommes a cependant abouti à 3 naissances $[7,4]$, suggérant que certains types d'anomalies de la pièce connective sont compatibles avec la formation de l'aster et du développement embryonnaire.

\section{LES PHÉNOTYPES PATHOLOGIQUES FLA- GELLAIRES MONOMORPHES}

Un large spectre d'anomalies ultrastructurales flagellaires a été décrit [revues dans 1 et 2]. Le flagelle étant à la base du mouvement des spermatozoïdes, l'asthénozoospermie sévère à totale est la manifestation la plus visible des anomalies des constituants du flagelle. Très schématiquement on distingue 2 groupes de phénotypes pathologiques flagellaires : la dysplasie de la gaine fibreuse (DFS), et la dyskinésie ciliaire primitive (DCP). Dans la DFS, l'arrangement des composants de la gaine fibreuse, des colonnes longitudinales et des rayons transverses est perturbé. La mobilité moyenne est très réduite avec une mobilité progressive quasi nulle. Un important pourcentage de spermatozoïdes avec des flagelles courts peut être observé. Dans la DCP, les spermatozoïdes sont immobiles, ont cependant une morphologie normale sur frottis coloré en microscopie optique. 
En microscopie électronique, les bras de dynéine sont absents, ainsi que, le plus souvent, les microtubules centraux et même l'axonème. Tous ces phénotypes ont souvent une incidence familiale et géographique, ce qui suggère une origine génétique. L'identification des gènes impliqués et des mutations est complexe car les phénotypes sont multiples.

Les hommes présentant une asthénozoospermie sévère, voire une akinétospermie, liée à un phénotype DFS ou DCP ont une infertilité primaire. En théorie, la microinjection du spermatozoïde dans l'ovocyte devrait permettre une fécondation et donc traiter ce type d'infertilité. Cependant, le succès de l'ICSI est totalement dépendant du choix du spermatozoïde vivant à microinjecter grâce au test hypo-osmotique, même si, la plupart du temps, la vitalité de ces spermatozoïdes est normale. L'obtention de grossesses et de naissances en ICSI est possible avec un taux moyen de fécondation supérieur à $50 \%[2,6]$. II semble cependant que le succès de l'ICSI soit influencé par le phénotype ; une identification précise des anomalies flagellaires en microscopie électronique est indispensable et constitue un facteur prédictif du succès de la micromanipulation.

\section{CONCLUSION}

Bien que rares, les phénotypes altérés monomorphes sont susceptibles d'être rencontrés dans tout laboratoire effectuant des spermogrammes. Les signes d'appel sont variables et divers. Ils peuvent correspondre à l'observation d'une tératozoospermie sévère touchant la plupart des spermatozoïdes : têtes rondes sans acrosome (globozoospermie), spermatozoïdes décapités et flagelles isolés mais mobiles (pinheads), flagelles courts (DFS). Le biologiste peut également être interpellé par des anomalies quantitatives et qualitatives de la mobilité des spermatozoïdes alors que les autres paramètres du spermogramme sont normaux. L'asthéno-zoospermie est généralement importante et même totale (akinétospermie) sans nécrospermie ni tératozoospermie dans les phénotypes de la DCP. II n'existe généralement pas de désordre andrologique associé, et les autres paramètres du sperme sont le plus souvent normaux, ce qui rend parfois difficile le dépistage de certains de ces phénotypes, en particulier ceux pour lesquels l'aspect des spermatozoïdes est strictement normal en microscopie optique.

Les spermatozoïdes doivent être étudiés en microscopie électronique pour connaître précisément et quantifier les structures altérées, car on sait que les phénotypes peuvent être très variables. L'absence de fertilité naturelle est quasiment constante et seule la micro-injection des spermatozoïdes dans l'ovocyte (ICSI) peut permettre dans certains cas d'obtenir une grossesse. Le succès de I'ICSI est extrêmement variable et dépend des altérations observées. De plus, aux défauts des organites peuvent s'associer des altérations de la structure de la chromatine et de l'intégrité de l'ADN, ce qui pourrait compromettre la fécondation et l'implantation des embryons. En effet, dans ces phénotypes, les taux de fécondation et de grossesse restent faibles en comparaison de ceux de I'ICSI en général.

Tout laisse penser, incidence familiale et géographique en particulier, que certains de ces phénotypes ont une origine génétique, bien que les gènes impliqués et les mutations n'aient pas encore été décrits. Par conséquent, il apparaît indispensable dans ces phénotypes de délivrer au couple une information génétique.

\section{REFERENCES}

1. CHEMES H.E. : Phenotypes of sperm pathology : genetic and acquired forms in infertile men. J. Androl., 2000, 6 : 799-808.

2. CHEMES H.E., RAWE V. : Sperm pathology : a step beyond descriptive morphology. Origin, characterisation and fertility potential of abnormal sperm phenotypes in infertile men. Hum. Reprod. Update, 2003, 5 : 405-428.

3. DAM A.H.D.M, FEENSTRA 1., WESTPHAL J.R., RAMOS L., VAN GOLDE R.J.T., KREMER J.A.M. : Globozoospermia revisited. Hum. Reprod. Update, 2007, $13: 63-75$.

4. EMERY B.R., THORP C., MALO J.W., CARRELL P.D. : Pregnancy from intracytoplasmic sperm injection of a sperm head and detached tail. Fertil. Steril., 2004, 81 : 686688.

5. LUNDIN K., SJOGREN A., NILSSON N., HAMBERGER L.: Fertilization and pregnancy after intracytoplasmic microinjection of acrosomeless spermatozoa. Fertil. Steril., 1994, $62: 1266-1267$.

6. MITCHELL V., RIVES N., ALBERT M. et al. : Outcome of ICSI with ejaculated spermatozoa in a series of men with distinct ultrastructural flagellar abnormalities. Hum. Reprod., 2006, 21, 8 : 2065-2074.

7. PORCU G., MERCIER G., BOYER P. et al. : Pregnancies after ICSI using sperm with abnormal head-tail junction from two brothers : case report. Hum. Reprod., 2003, 18 : 562-567.

8. RYBOUCHKIN A.V., VAN DER STRATEN F., QUATACKER J., DE SUTTER P., DHONDT M. : Fertilization and pregnancy after assisted oocyte activation and intracytoplasmic sperm injection in a case of round-headed sperm associated with deficient oocyte activation capacity. Fertil. Steril., 1997, $68: 1144-1147$.

9. SCHIRREN C.G., HOLSTEIN A.F., SCHIRREN C. : Uber die morphogenese rundköpfiger spermatozoen des menschen. Andrology, 1971, $3: 117-125$.

Manuscrit reçu : septembre 2007 ; accepté septembre 2007. Communication au XXVlème Congrès de la SALF, décembre 2007, Colmar 